HNO 2020 68 (Suppl 2):S86-S92 https://doi.org/10.1007/s00106-020-00824-1 Published online: 26 March 2020 (C) The Author(s) 2020

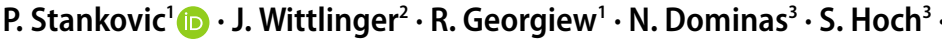 \\ T. Wilhelm ${ }^{1,4}$ iD \\ ' Department of Otolaryngology, Head/Neck \& Facial Plastic Surgery, Sana Kliniken Leipziger Land, Borna, \\ Germany \\ ${ }^{2}$ Department of Otolaryngology, Head and Neck Surgery, Martin Luther-University Halle-Wittenberg, \\ Halle (Saale), Germany \\ ${ }^{3}$ Department of Otolaryngology, Philipps-University Marburg, Marburg, Germany \\ ${ }^{4}$ Medical Faculty, Philipps-University Marburg, Marburg, Germany
}

\title{
Continuous intraoperative neuromonitoring (CIONM) in head and neck surgery-a review
}

devised facial nerve stimulators for use in parotid and ear surgery $[10,20]$.

In recent decades, the use of IONM has become standard in many institutions where it is used mainly as an identifying tool during careful dissection in the proximity of the nerve. The main surgical fields in which it is employed are surgeries on the thyroid gland where the vagus nerve and recurrent laryngeal nerve are monitored, parotidectomy, and surgery of the posterior cranial fossa where the facial nerve is monitored.

During the course of the operation, the surgeon uses a stimulator probe in order to identify the nerve and differentiate it from other tissue. The probe is placed onto the nerve, thus closing a circuit and producing further visual or acoustic stimuli every time the nerve is touched. Such application can be defined as intermittent IONM (iIONM). tus, Krause stimulated the facial nerve and visually noted "... contractions of the facial region, especially of the orbicularis oculi, as well as the branches supplying the nose and the mouth." The breakthrough years for IONM came in the 1960s when Flisberg and Lindholm [7] introduced it to thyroid surgery, and Parsons and Hilger, in different studies,

The German version of this article can be found under https://doi.org/10.1007/s00106020-00823-2.

\section{) Modern IONM has prognostic implications for intraoperative information}

In otolaryngology, iIONM is widespread in parotid surgery. Some authors have reported lower postoperative facial palsy rates when using iIONM [16, 27, 35]. On the other hand, there are studies that state that iIONM does not lead to less paresis after surgery $[4,9,40]$. In the United States, there is no clear recom- mendation on whether a head and neck surgeon should use iIONM when performing surgeries on the parotid gland. As a result, $40 \%$ of otolaryngologists in United States do not currently routinely use iIONM, but rely rather on their surgical skills and knowledge of anatomical landmarks [17]. A recent metaanalysis comparing parotidectomies with and without iIONM found the incidence of immediate postoperative facial nerve palsy following parotidectomy while using iIONM to be significantly lower when compared with that of no intraoperative monitoring [34].

\section{1) The aim of clONM is to provide real-time monitoring of the nerve status during exposure}

Recently, the development of IONM has been enhanced by the establishment of so-called continuous intraoperative neuromonitoring (cIONM) in clinical practice. This aims to provide realtime monitoring of the nerve status during the entire course of exposure and, perhaps more importantly, to predict postoperative function. This is done either actively (acIONM) or passively (pcIONM; - Table 1). During acIONM, the nerve is stimulated continuously during the whole procedure. This can be facilitated using either an electrode that is placed on the nerve or near the 


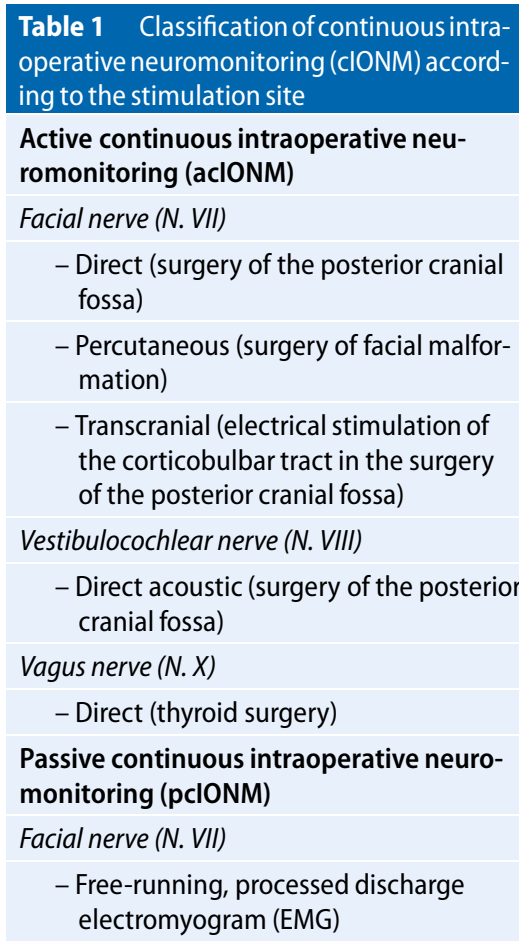

Vestibulocochlear nerve (N. VIII)

- Direct acoustic (surgery of the posterio cranial fossa)

Vagus nerve (N.X)

- Direct (thyroid surgery)

Passive continuous intraoperative neuromonitoring (pcIONM)

Facial nerve (N. VII)

- Free-running, processed discharge electromyogram (EMG)

nerve, by transcranial nerve stimulation or, in the case of vestibulocochlear monitoring, using acoustic stimuli at a given frequency $(\mathrm{Hz})$. Active cIONM has been developed in thyroid surgery $[5,8,12,14,21,25,30,32,33]$, surgery on the posterior cranial fossa $[1,2,37$, 41 ], and surgery on vascular anomalies [38] where vagus, facial, and vestibulocochlear nerves are at risk.

It is important, at this point, to differentiate between iIONM and cIONM, because many authors refer to iIONM as continuous, thus resulting in some confusion. During iIONM, the patient is indeed continuously "attached" to the monitoring device, but the information that the surgeon receives is in fact not continuous. In this setting, only the identification of the nerve from the surrounding tissue is possible and only when the surgeon actively makes use of the stimulator probe. The nerve amplitudes and latencies are not subject to analysis. This means that for the majority of intraoperative time "what the nerve has to say is not heard." During cIONM, on the other hand, uninterrupted analysis of amplitude and latency of the nerve is "fed" into a monitoring device, enabling computerized analysis.

\section{The benefits of cIONM}

\section{aclONM of the vagus nerve in thyroid surgery}

Five different modalities of acIONM are described in the literature (- Table 1). Active cIONM is facilitated by an electrode placed on the vagus nerve between the common carotid artery and internal jugular vein and is used to monitor the functioning of the recurrent vagus nerve. The nerve has to be dissected from the carotid sheet and have $360^{\circ} \mathrm{ex}$ posure in order to position the electrode. Here, a pattern of impending nerve injury has been identified [32]. It has been noted that decreases of amplitude or latency alone do not have a prognostic factor. However, a combination of a deterioration of amplitude by more than $50 \%$ and prolonged latency by more than $10 \%$, namely, multiple combined events (mCE), precedes complete loss of signal (LOS; decline of the amplitude to less than $100 \mu \mathrm{V})$ and thus predicts postoperative vocal cord palsy (VCP; - Fig. 1).

When the mCE does not evolve to an LOS, the postoperative function of the nerve remains normal. Therefore, when a surgeon notes $\mathrm{mCE}$ during surgery and performs an avoidance maneuver, for example, reduced traction, postoperative palsy can be avoided. This is a novel method of avoiding impending nerve injury.

\section{I) During ilONM, most of the time "what the nerve has to say is not heard"}

Bearing in mind that a neurophysiological pattern of nerve injury can be seen during cIONM, a study was published that compared cIONM and IIONM in 1526 consecutive patients undergoing thyroid surgery, split into groups of a similar size [33]. Continuous IONM demonstrated a statistically significant difference with regard to permanent VCP: There were no cases of permanent VCP in the cIONM group whereas four cases of permanent VCP were noted in the iIONM group. This study showed an important benefit of cIONM: In the
cIONM patients observed, 77 mCEs were noted, 63 of which (82\%) could be actively reversed by the surgeon by stopping the suspected causative maneuver [33].

Furthermore, Schneider et al. demonstrated in their prospective, multicentric study an excellent prognosis for postoperative vocal fold function relative to vagus amplitude recovery of $\geq 50 \%$ after LOS [31]. In bilateral thyroid resection, this information helps decision-making regarding whether the other side should be resected or not when LOS is encountered on the first side. If the LOS recovers to $\geq 50 \%$ of the baseline amplitude, the resection on the contralateral side can be performed safely.

\section{Direct acIONM in posterior cranial fossa surgery}

Direct stimulation of the facial nerve during posterior cranial fossa surgery was described by Amano [1]. The author used a ball-type electrode to directly stimulate the root exit zone of the nerve and secured it with cotton pads. Significant differences according to the House-Brackmann (HB) grade regarding the last maximal amplitude as well as the amplitude preservation ratio (last amplitude at the end of resection compared with the baseline amplitude) were noted between the groups of different facial nerve palsy. Furthermore, it was reported that patients with good postoperative functional results according to the $\mathrm{HB}$ grade, along with patients having long-term postoperative improvement in HB grade, showed statistically higher amplitude preservation rates [1]. Therefore, acIONM was demonstrated to be potentially useful in surgery of the posterior cranial fossa with regard to the facial nerve.

\section{acIONM in vascular malformation surgery}

Another extracranial method of facial nerve neuromonitoring is percutaneous stimulation. Ulkatan and colleagues used two monopolar EMG needles during surgery on facial vascular malformations, introducing them percutaneously toward the stylomastoid foramen [38]. 
The electrodes were used as stimulator probes for preoperative percutaneous mapping of the facial nerve, acIONM, and intraoperative mapping of the facial nerve. In 161 mostly young patients (mean age: $14 \pm 14$ years), acIONM enabled preoperative nerve mapping, mainly in patients where face edema due to preoperative sclerotherapy masked muscle twitches; needle placement in all surgeries was achieved without complications [38]. A baseline value of the compound muscle action potential (CMAP) was set at the beginning of the surgery and values of $<50 \%$ CMAP alerted the surgeon to stop the manipulation until the amplitude normalized. Intraoperative nerve injury was correctly recognized in all three cases and a direct end-to-end neurorrhaphy was performed, enabling a long-term recovery that reached HB grade I/II in these patients.

\section{acIONM in transcranial stimulation during posterior fossa surgery}

Multipulse transcranial electric stimulation (TES) of the corticobulbar pathway during posterior cranial fossa surgery is a method of continuous monitoring of the functioning of the facial nerve through analysis of the muscle motor evoked potential (FNMEP). The stimulator, in the form of a cup electrode, is placed above the skull. This method uses clusters of three to four pulses of current, producing a supramaximal stimulation (100-400 V) with an interpulse interval of $1-2 \mathrm{~ms}$ and a cluster frequency of $5.6-3.3 \times 10^{-3} \mathrm{~Hz}$ [6]. In a study by Dong et al., no patient with a final amplitude of $50 \%$ or greater than the baseline amplitude had more than a mild deterioration in facial nerve function when compared with their preoperative facial nerve function [6].

\section{acIONM of the vestibulocochlear nerve during posterior fossa surgery}

Neuromonitoring of the vestibulocochlear nerve using brain stem auditory evoked potentials (BAEP) during cerebellopontine angle surgery can also be described as acIONM. During the prepa- ration, acoustic clicks of $100-110 \mathrm{~dB}$ are continuously delivered to the ear in the proximity of the eighth cranial nerve by means of ear pods. JEWETT waves I and $\mathrm{V}$ deliver the most useful information owing to their constancy. To some extent, wave III can also be used for interpretation. The other ear receives white noise of $60-70 \mathrm{~dB}$ in order to mask the contralateral clicks. A similar method used in the same type of surgery is electrocochleography (ECochG) acIONM, which obtains a waveform equivalent to JEWETT wave I of the BAEP, however with significantly higher amplitude. Here, compound action potential (CAP) should also be noted. This method takes advantage of an electrode placed either between the tumor and the root entry of the nerve into the brain stem [41] or distal to the tumor [11]. The BAEP, ECochG, and CAP are complementary methods that do not exclude one another but, on the contrary, are commonly used simultaneously.

The BAEP acIONM has shown reliable results in predicting postoperative hearing function. For example, Neu [19] stratified patients monitored by acIONM using BAEP into four groups. All patients with stable wave $\mathrm{V}$ (pattern 1) showed definite hearing preservation while all patients with irreversible abrupt loss of BAEP (pattern 2) lost their hearing, despite early hearing preservation in two cases. All patients with irreversible progressive loss of either wave I or wave $\mathrm{V}$ (pattern 3) eventually suffered from definite postoperative hearing loss, despite early hearing preservation in two cases. Those cases with intraoperative reversible loss of BAEP (pattern 4) showed variable short- and long-term hearing outcomes [19]. In a study by Yamakami, BAEP and CAP were used concomitantly [41]. Reliable BAEP values referring to wave $\mathrm{V}$ could be obtained only in $41 \%$ of patients, whereas reproducible CAP without artifacts were noted in $91 \%$ of patients. All patients who preserved CAP on completion of a microsurgical tumor removal preserved serviceable hearing postoperatively, showing a $100 \%$ specificity and sensitivity [41].
HNO 2020 - 68 (Suppl 2):S86-S92

https://doi.org/10.1007/s00106-020-00824-1

(c) The Author(s) 2020

P. Stankovic · J. Wittlinger - R. Georgiew · N. Dominas $\cdot$ S. Hoch · T. Wilhelm

Continuous intraoperative neuromonitoring (CIONM) in head and neck surgery-a review

\section{Abstract}

Although the history of intraoperative neuromonitoring (IONM) dates back to the 19th century, the method did not evolve further than the mere differentiation of nerves until recently. Only the development of continuous IONM (cIONM) has allowed for non-stop analysis of excitation amplitude and latency during surgical procedures, which is nowadays integrated into the software of almost all commercially available neuromonitoring devices. The objective of cIONM is real-time monitoring of nerve status in order to recognize and prevent impending nerve injury and predict postoperative nerve function. Despite some drawbacks such as false-positive/negative alarms, technical artefacts, and rare adverse effects, clONM remains a good instrument which is still under development. Active (acIONM) and passive (pclONM) methods of cIONM are described in literature. The main fields of cIONM implementation are currently thyroid surgery (in which the vagal nerve is continuously stimulated) and surgery to the cerebellopontine angle (in which the facial nerve is either continuously stimulated or the discharge signal of the nerve is analyzed via pcIONM). In the latter surgery, continuous monitoring of the cochlear nerve is also established.

\section{Keywords}

Cerebellopontine angle - Thyroidectomy . Intraoperative neurophysiological monitoring · Facial nerve - Vagal nerve

\section{pcIONM of the facial nerve during posterior fossa surgery}

In contrast to acIONM, methods of continuous monitoring that can be described as passive cIONM have evolved. These rely purely on analyzing the discharge patterns that occur during the operation. Such "free-running EMG" is used in neurosurgery in the monitoring of the facial nerve. In 1986, Prass and Lüders described spikes, bursts, and three types of 


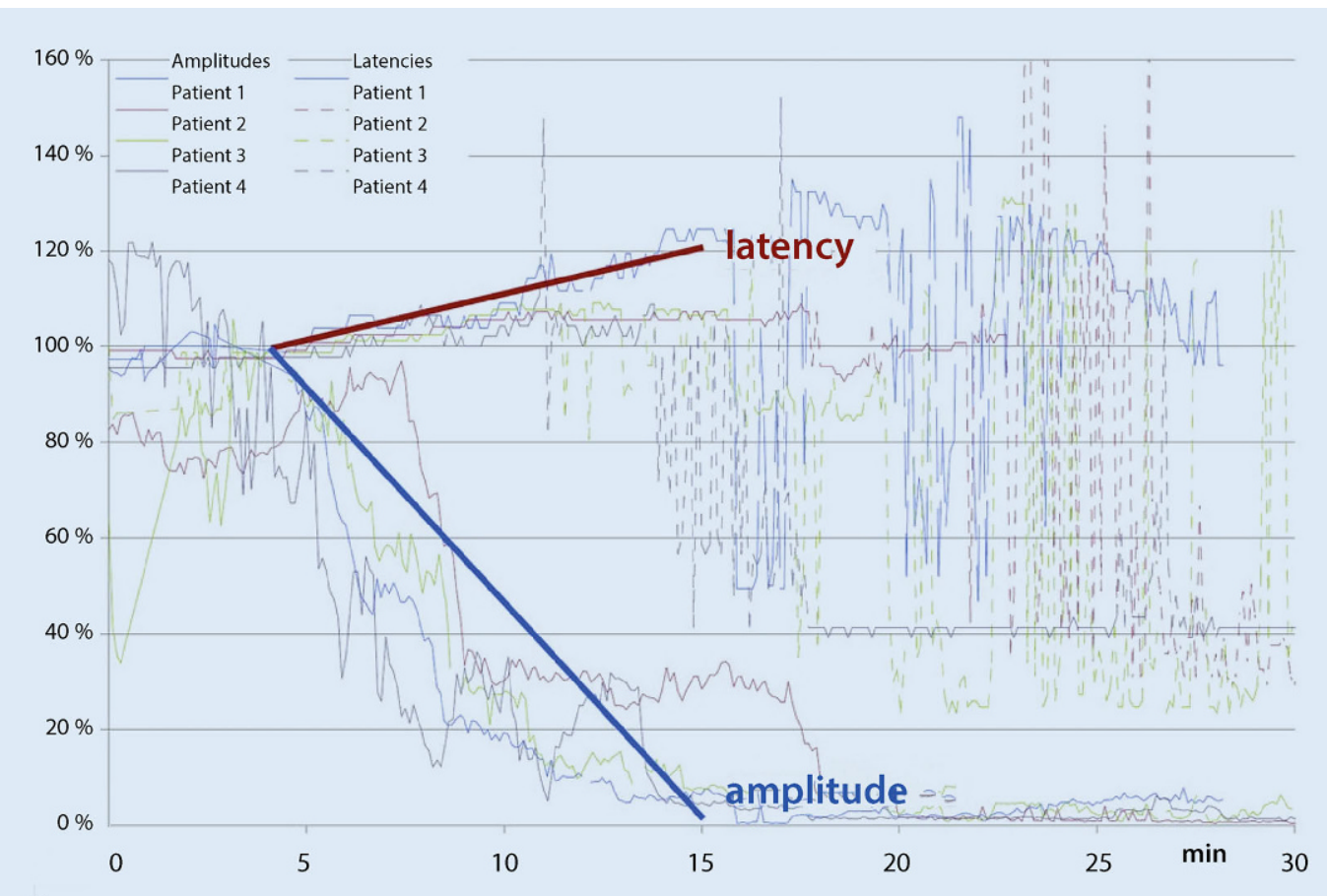

a

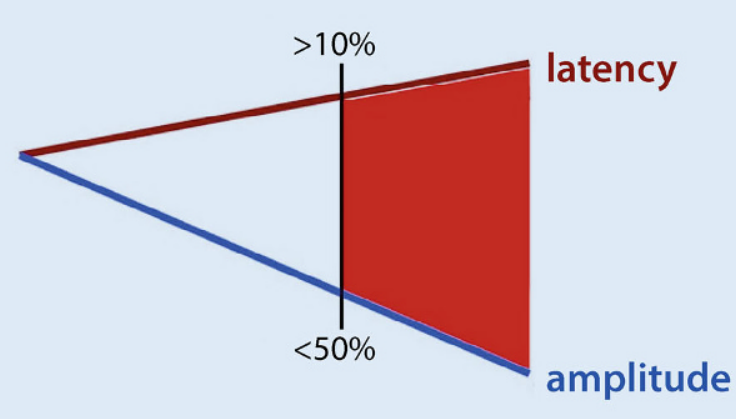

Fig. $1<$ a Complete loss of signal during active continuous intraoperative neuromonitoring of the vagus in thyroid surgery resulting in postoperative recurrent laryngeal nerve palsy. b Schematic cut-offs for postoperative impaired nerve function. (From [32]. Reprinted with permission (c) John Wiley and Sons)

trains in the EMG signal during posterior fossa surgery on 30 patients [22]. Trains represented sustained periodic EMG activity that lasted for seconds. The presence of A-trains [22], roughly referring to high-frequency and low-amplitude sinusoidal EMG patterns, were later correlated with a lower postoperative $\mathrm{HB}$ score [26]. Prell processed the train time in the signal obtained on a computer in an automated fashion offline [24]. This evolved into software that was used in an online fashion in the operating theater [23]. The software enabled a real-time quantification of train time, informing the surgeon on the "cumulative damage" to the nerve [23]. By having real-time information on the impending nerve injury, the surgeon could estimate the probable postoperative nerve function and actively change the operative strategy in order to avoid further deterioration of the nerve function.

\section{》) Signal recovery of $\geq 50 \%$ after LOS correlates with normal postoperative nerve function}

The information the surgeon obtained was represented in analogy to a traffic light. The status of the nerve remained in the "green area" when the train time remained under $0.125 \mathrm{~s}$, which meant the dissection could be continued safely. When the train time exceeded $0.125 \mathrm{~s}$ but remained under $2.5 \mathrm{~s}$ the light was changed to "orange," which indicated the need for increased care because this amount of train time accounted for a deterioration in the HB scale to the third grade in $25 \%$ of patients with normal pre- operative facialnerve function. An excess of train time beyond $2.5 \mathrm{~s}$ resulted in the light changing to "red" and was clearly associated with a significant increase in paresis, which prompted the surgeon to abort the manipulation and re-evaluate the surgical plan. For example, the angle and site of further preparation was changed, nimodipine was applied intraoperatively, resection was stopped in selected patients, and a revision procedure was scheduled.

\section{Safety of cIONM}

The safety of cIONM is of paramount importance. Passive cIONM can be excluded from precaution for obvious reasons: This method relies on the pure analysis of the signal that is noted and no active stimulation takes place, thus 
no nerve damage could possibly be done. This is not the case with acIONM where active stimulation is applied. The fact that one involves a novel method using active stimulation to the nerve makes the issue of safety of outmost importance.

Active cIONM has generally been described as a safe procedure in thyroid surgery throughout high-volume studies $[8,32,33]$. The safety of acIONM was demonstrated in a prospective study in which no heart rate variability and immunomodulatory effects were noted in spite of continuous stimulation of the vagus [8]. This was also observed in an earlier study by the same group of authors, where once again the distinct influence of acIONM on the autonomous nervous system balance was applied without alterations to heart rate, rhythm, or hemodynamic parameters [39]. In a study involving 102 patients, Phelan noted neither cases of adverse amplitude or latency changes, nor cases of adverse gastric, cardiac, pulmonary, or gastrointestinal side effects [21]. Active cIONM has been carried out safely also in patients with advanced atrioventricular block [28].

In an experimental study of 13 pigs by Lee, acIONM was applied using automated period stimulation in order to examine the force needed for a traction injury of the recurrent laryngeal nerve [15]. The nerves were deliberately stretched until loss of signal occurred. Seven days after the experiment, all nerves displayed EMG signal recovery, thus showing that acIONM alone does not induce structural damage to the nerve [15] and that recovery will take place.

However, some adverse effects have been reported $[3,18,36]$. In one patient, reversible vagal neuropraxia with visible perineural ecchymosis was caused by the APS $^{\circledR}$ stimulating electrode (Automatic Periodic Stimulation, Medtronic, Jacksonville, FL, USA), as well as a subsequent inability to stimulate the nerve after the event. A short-term postoperative palsy was noted with reversal after 1 month. In the same publication, an allegedly serious hemodynamic instability (bradycardia and hypotension) was noted after the onset of acIONM in a young healthy patient with no history of cardiac events. The effect could be promptly reversed by removing the electrode. Upon replacement of the electrode, the exact same effect was again noted, and again was rapidly reversed by removing the electrode once more. Patient recruitment in the study was abandoned after these two adverse events [36].

Two studies together comprising approximately 250 nerves at risk reported an identical risk of $2 \%$ for vagus nerve injury due to the placement of the APS ${ }^{\circledR}$ electrode $[3,18]$. The events were not associated with permanent postoperative nerve palsy.

\section{Safe application of acIONM in children}

There are also studies regarding the safety of acIONM in children. For example, Bozinov used transcranial electric stimulation to continuously monitor FNMEP in 21 patients (median age: 5.5 years; range: 5 months to 15 years). The FNMEP was also feasible and safe in the young population, with similar values achieved in the prediction of postoperative facial nerve function to those of adults reported in other studies. The $\mathrm{HB}$ grade remained the same pre- and postoperatively in 23 of 24 surgeries. The presence of FNMEP influenced the surgical strategy and contributed to tumor resection in those cases where direct nerve stimulation gave no muscle response [2]. A second example of acIONM in children is the previously mentioned study by Ulkatan where percutaneous stimulation was applied in 201 surgeries on 161 patients aged $14 \pm 14$ years [38]. Safe application of acIONM in thyroid surgery was demonstrated in a largescale study including 105 children [29].

\section{Limitations of cIONM}

Aside from the aforementioned rare adverse effects reported due to electrode placement, some other limitations come to mind. In reference to our own experience with neuromonitoring, one must note the problem of technical artifacts and false-positive or false-negative alarms that often occur. The field of cIONM, with real-time nerve surveillance, emphasizes the burden of such false alarms. All the aforementioned studies share the same problem of a low positive predictive value. In this context, for example, in thyroid surgery, a falsepositive alarm can be responsible for unnecessarily delaying the procedure of the contralateral side.

\section{) To date, no studies have applied cIONM in parotid surgery}

Apart from rare retrospective studies comparing cIONM with IIONM in thyroid surgery, there are no studies comparing this novel method with the conventional iIONM in the other fields of application mentioned earlier. Prospective randomized studies are urgently needed in order to prove the claimed benefits.

Some of the methods described remain "single-study reports," meaning that the method failed to become establish in clinical practice(see Sects. "Direct acIONM in posterior cranial fossa surgery" and "acIONM in vascular malformation surgery"). In some studies, only the last acquired amplitude and the baseline amplitude were used for statistical evaluation (see Sect. "Direct acIONM in posterior cranial fossa surgery"), in others the frequency of continuous stimulation was only one in 3-5 min (see Sect. "acIONM in transcranial stimulation during posterior fossa surgery"), questioning the need for continuous stimulation.

\section{Practical conclusion}

The novel methods of continuous
intraoperative neuromonitoring
(cIONM) have made the recognition
of impending nerve injury and the
consequent change of operative
strategy possible.
Furthermore, the prediction of post-
operative nerve function based on
clONM has been refined in compari-
son to that of intermittent intraoper-
ative neuromonitoring (ilONM).
Continuous IONM brings a new
dimension to the field of neuromoni-
toring.


- It is a new evolving instrument destined to help surgeons in performing surgical maneuvers in close proximity to neural structures.

- It cannot and does not replace good operative technique and patency; however, it does provide reliable and safe assistance.

- The safety of active cIONM (acIONM) has been demonstrated in animal and human studies.

- The field appears to be open for future studies, especially in surgery of the parotid gland where to date neither acIONM nor pcIONM have been applied.

\section{Corresponding address}

\section{Professor T. Wilhelm, MD PhD}

Department of Otolaryngology, Head/Neck \& Facial Plastic Surgery, Sana Kliniken Leipziger Land

Rudolf-Virchow-Straße 2, 04552 Borna,

Germany

thomas.wilhelm@sana.de

Funding. This project was funded entirely by the Department of Otolaryngology, Head/Neck \& Facial Plastic Surgery, Sana Kliniken Leipziger Land, Germany.

Funding. Open Access funding provided by Projekt DEAL.

\section{Compliance with ethical guidelines}

Conflict of interest. P. Stankovic, J. Wittlinger, R. Georgiew, N. Dominas, S. Hoch and T. Wilhelm declare that they have no competing interests.

For this article no studies with human participants or animals were performed by any of the authors. All studies performed were in accordance with the ethical standards indicated in each case.

The supplement containing this article is not sponsored by industry.

Open Access. This article is licensed under a Creative Commons Attribution 4.0 International License, which permits use, sharing, adaptation, distribution and reproduction in any medium or format, as long as you give appropriate credit to the original author(s) and the source, provide a link to the Creative Commons licence, and indicate if changes were made.

The images or other third party material in this article are included in the article's Creative Commons licence, unless indicated otherwise in a credit line to the material. If material is not included in the article's Creative Commons licence and your intended use is not permit- ted by statutory regulation or exceeds the permitted use, you will need to obtain permission directly from the copyright holder.

To view a copy of this licence, visit http:// creativecommons.org/licenses/by/4.0/.

\section{References}

1. Amano $M$, Kohno $M$, Nagata $O$ et al (2011) Intraoperative continuous monitoring of evoked facial nerve electromyograms in acoustic neuroma surgery. Acta Neurochir 153:1059-1067 (discussion 1067)

2. Bozinov O, Grotzer MA, Sarnthein J (2015) Intraoperative monitoring of facial nerve motorevoked potentials in children. World Neurosurg 84:786-794

3. Brauckhoff K, Vik R, Sandvik L et al (2016) Impact of EMG changes in continuous vagal nerve monitoring in high-risk endocrine neck surgery. World J Surg 40:672-680

4. Deneuve S, Quesnel S, Depondt J et al (2010) Management of parotid gland surgery in a university teaching hospital. Eur Arch Otorhinolaryngol 267:601-605

5. Deniwar A, Kandil E, Randolph G (2015) Electrophysiological neural monitoring of the laryngeal nerves in thyroid surgery: review of the current literature. Gland Surg 4:368-375

6. Dong C, Macdonald D, Akagami R et al (2005) Intraoperative facial motor evoked potential monitoring with transcranial electrical stimulation during skull base surgery. Clin Neurophysiol 116:588-596

7. Flisberg K, Lindholm T (1969) Electrical stimulation of the human recurrent laryngeal nerve during thyroid operation. Acta Otolaryngol Suppl 263:63-67

8. Friedrich C, Ulmer C, Rieber F et al (2012) Safety analysis of vagal nerve stimulation for continuous nerve monitoring during thyroid surgery. Laryngoscope 122:1979-1987

9. Grosheva M, Klussmann JP, Grimminger $C$ et al (2009) Electromyographic facial nerve monitoring during parotidectomy for benign lesions does not improve the outcome of postoperative facial nerve function: a prospective two-center trial. Laryngoscope 119:2299-2305

10. Hilger JA (1964) Facial nerve stimulator. Trans Am Acad Ophthalmol Otolaryngol 68:74-76

11. Jackson L, Robertson J (2000) Acoustic neuroma surgery: use of cochlear nerve actionpotential monitoring for hearing preservation. Am J Otolaryngol 21:249-259

12. Jonas J (2010) Continuous vagal nerve stimulation for recurrent laryngeal nerve protection in thyroid surgery. Eur Surg Res 44:185-191

13. Krause $F$ (1907) Chirurgie des Gehirns und Rückenmarks. Rebman, Berlin

14. Lamade W, Ulmer C, Friedrich C et al (2011) Signal stability as key requirement for continuous intraoperative neuromonitoring. Chirurg 82:913-920

15. Lee HY, Cho YG, You JY et al (2016) Traction injury of the recurrent laryngeal nerve: results of continuous intraoperative neuromonitoring in a swine model. Head Neck 38:582-588

16. Lopez M, Quer M, Leon X et al (2001) Usefulness of facial nerve monitoring during parotidectomy. Acta Otorrinolaringol Esp 52:418-421
17. Lowry TR, Gal TJ, Brennan JA (2005) Patterns of use of facial nerve monitoring during parotid gland surgery. Otolaryngol Head NeckSurg 133:313-318

18. Arteaga MA, Peloni G, Leuchter I et al (2018) Modification of the surgical strategy for the dissection of the recurrent laryngeal nerve using continuous Intraoperative nerve monitoring. World J Surg 42:444-450

19. Neu M, Strauss C, Romstöck J et al (1999) The prognostic value of intraoperative BAEP patterns in acoustic neurinoma surgery. Clin Neurophysiol 110:1935-1941

20. Parsons RC (1968) Electrical nerve stimulation at surgery. Laryngoscope 78:742-748

21. Phelan E, Schneider R, Lorenz K et al (2014) Continuous vagal IONM prevents recurrent laryngeal nerve paralysis by revealing initial EMG changes of impending neuropraxic injury: a prospective, multicenter study. Laryngoscope 124:1498-1505

22. Prass RL, Luders H (1986) Acoustic (loudspeaker) facial electromyographic monitoring: Part 1. Evoked electromyographicactivity during acoustic neuroma resection. Neurosurgery 19:392-400

23. Prell J, Rachinger J, Scheller C et al (2010) A real-time monitoring system for the facial nerve. Neurosurgery 66:1064-1073 (discussion 1073)

24. Prell J, Rampp S, Romstöck J et al (2007) Train time as a quantitative electromyographic parameter for facial nerve function in patients undergoing surgery for vestibular schwannoma. J Neurosurg 106:826-832

25. Randolph GW, Dralle H, International Intraoperative Monitoring Study Group et al (2011) Electrophysiologic recurrent laryngeal nerve monitoring during thyroid and parathyroid surgery: international standards guideline statement. Laryngoscope 121(Suppl 1):S1-S16

26. Romstöck J, Strauss C, Fahlbusch R (2000) Continuous electromyography monitoring of motor cranial nerves during cerebellopontine angle surgery. J Neurosurg 93:586-593

27. Savvas E, Hillmann S, Weiss D et al (2016) Association between facial nerve monitoring with postoperative facial paralysis in parotidectomy. JAMA Otolaryngol Head Neck Surg 142:828-833

28. Schneider R, Machens A, Bucher Metal (2016) Continuous intraoperative monitoring of vagus and recurrent laryngeal nerve function in patients with advanced atrioventricular block. Langenbecks Arch Surg 401:551-556

29. Schneider R, Machens A, Sekulla C et al (2018) Twenty-year experience of paediatric thyroid surgery using intraoperative nerve monitoring. $\mathrm{Br}$ JSurg 105:996-1005

30. Schneider R, Przybyl J, Hermann M et al (2009) A new anchor electrode design for continuous neuromonitoring of the recurrent laryngeal nerve by vagal nerve stimulations. Langenbecks Arch Surg 394:903-910

31. Schneider R, Randolph G, Dionigi G et al (2019) Prediction of postoperative vocal fold function after Intraoperative recovery of loss of signal. Laryngoscope 129:525-531

32. Schneider R, Randolph GW, Sekulla C et al (2013) Continuous intraoperative vagus nerve stimulation for identification of imminent recurrent laryngeal nerve injury. Head Neck 35:1591-1598

33. Schneider R, Sekulla C, Machens A et al (2015) Postoperative vocal fold palsy in patients undergoing thyroid surgery with continuous or intermittent nerve monitoring. Br JSurg 102:1380-1387

34. Sood AJ, Houlton JJ, Nguyen SA et al (2015) Facial nerve monitoring during parotidectomy: a sys- 


\section{Review articles}

tematic review and meta-analysis. Otolaryngol Head Neck Surg 152:631-637

35. Terrell JE, Kileny PR, Yian C et al (1997) Clinical outcome of continuous facial nerve monitoring during primary parotidectomy. Arch Otolaryngol Head Neck Surg 123:1081-1087

36. Terris DJ, Chaung K, Duke WS (2015) Continuous vagal nerve monitoring is dangerous and should not routinely be done during thyroid surgery. World J Surg 39:2471-2476

37. Tokimura H, Sugata S, Yamahata H et al (2014) Intraoperative continuous monitoring of facial motor evoked potentials in acoustic neuroma surgery. Neurosurg Rev 37:669-676

38. Ulkatan S, Waner M, Arranz-Arranz B et al (2014) New methodology for facial nerve monitoring in extracranial surgeries of vascular malformations. Clin Neurophysiol 125:849-855

39. Ulmer C, Friedrich C, Kohler A et al (2011) Impact of continuous intraoperative neuromonitoring on autonomic nervous system during thyroid surgery. Head Neck 33:976-984

40. Witt R (1998) Facial nerve monitoring in parotid surgery: the standard of care? Otolaryngol Head NeckSurg 119:468-470

41. Yamakami I, Yoshinori H, Saeki N et al (2009) Hearing preservation and intraoperative auditory brainstemresponseand cochlearnervecompound action potential monitoring in the removal of small acoustic neurinoma via the retrosigmoid approach. J Neurol Neurosurg Psychiatry $80: 218-227$ 